This item was submitted to Loughborough's Research Repository by the author.

Items in Figshare are protected by copyright, with all rights reserved, unless otherwise indicated.

\title{
Hysteresis of the contact angle of a meniscus inside a capillary with smooth, homogeneous solid walls
}

\section{PLEASE CITE THE PUBLISHED VERSION}

http://dx.doi.org/10.1021/acs.langmuir.6b00721

\section{PUBLISHER}

(c) American Chemical Society

\section{VERSION}

AM (Accepted Manuscript)

\section{PUBLISHER STATEMENT}

This work is made available according to the conditions of the Creative Commons Attribution-NonCommercialNoDerivatives 4.0 International (CC BY-NC-ND 4.0) licence. Full details of this licence are available at: https://creativecommons.org/licenses/by-nc-nd/4.0/

\section{LICENCE}

CC BY-NC-ND 4.0

\section{REPOSITORY RECORD}

Kuchin, I., and Victor Starov. 2016. "Hysteresis of the Contact Angle of a Meniscus Inside a Capillary with Smooth, Homogeneous Solid Walls". Loughborough University. https://hdl.handle.net/2134/22274. 


\title{
Hysteresis of contact angle of a meniscus inside a capillary with smooth homogeneous solid walls
}

\author{
Igor V. Kuchin ${ }^{1}$, Victor M. Starov ${ }^{2 *}$ \\ ${ }^{1}$ Institute of Physical Chemistry and Electrochemistry, Russian Academy of Sciences, \\ Leninsky pr, 31/4, Moscow, 119071, Russia \\ ${ }^{2}$ Department of Chemical Engineering, Loughborough University, LE11 3TU, UK
}

\begin{abstract}
A theory of contact angle hysteresis of a meniscus inside thin capillaries with smooth, homogeneous solid walls is developed in terms of surface forces (disjoining/conjoining pressure isotherm) using quasi-equilibrium approach. The disjoining/conjoining pressure isotherm includes electrostatic, intermolecular and structural components. The values of the static receding, $\theta_{r}$, advancing, $\theta_{a}$, and equilibrium, $\theta_{e}$ contact angles in thin capillaries were calculated based on the shape of disjoining/conjoining pressure isotherm. It was shown that both advancing and receding contact angles depend on the capillary radius. The suggested mechanism of the contact angle hysteresis has a direct experimental confirmation: the process of receding is accompanied by formation of thick $\beta$ films on the capillary walls. The effect of transition from partial to complete wetting in thin capillaries is predicted and analyzed. This effect takes place in very thin capillaries, when the receding contact angle decreases to zero.
\end{abstract}

Keywords: wetting, contact angle hysteresis, disjoining/conjoining pressure, capillary meniscus.

\section{Introduction}

It is usually believed that static hysteresis of the contact angle is determined by the surface roughness and/or heterogeneity ${ }^{1}$. No doubt that a roughness and/or a chemical heterogeneity of the solid substrate contribute substantially to the contact angle hysteresis. It is assumed in this case that at each point of the surface the equilibrium value of the contact angle is established depending only on the local properties of the substrate. As a result a whole series of local thermodynamic

\footnotetext{
*Corresponding author: V.M.Starov@lboro.ac.uk
} 
equilibrium states can be realized, corresponding to a certain interval of contact angles. The maximum possible value corresponds to the static advancing contact angle, $\theta_{a}$, and the minimum possible value corresponds to the static receding contact angle, $\theta_{r}$. Hence, the dependency of the contact angle on the velocity of motion of a meniscus or a drop can be qualitatively described by the dependency presented by solid lines in Fig.1.

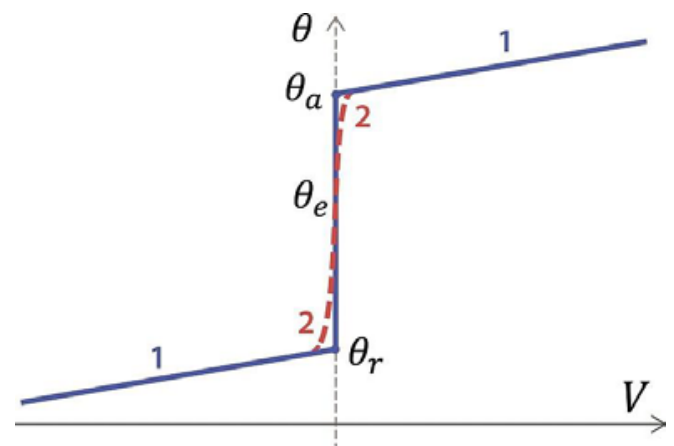

Fig. 1. Solid lines 1 - Idealized dependency of the contact angle on the advancing $(V>0)$ or the receding $(\mathrm{V}<0)$ velocity of a droplet/meniscus. All contact angles, $\theta$, between static advancing contact angle, $\theta_{a}$, and static receding contact angle, $\theta_{r}$, are considered as equilibrium contact angles. Curve 2 -0.0 a real dependency of the contact angle on the advancing or receding velocities. At any deviation from the equilibrium contact angle, $\theta_{e}$, the liquid drop/meniscus is in the state of a slow "microscopic motion", which almost abruptly transforms into "a macroscopic motion". In this case static advancing and receding contact angles, $\theta_{a}$ and $\theta_{r}$, are extrapolations to zero velocity.

However, roughness and/or heterogeneity of the surface are apparently not the sole reasons for contact angle hysteresis. There have been an increasing number of publications over the last years which confirmed the presence of contact angle hysteresis even on smooth, homogeneous surfaces ${ }^{2-7}$. However, the most convincing evidence for the presence of the above mentioned phenomenon is its presence on free liquid films ${ }^{8-12}$ : in this case there is hysteresis of a meniscus, which is located on thin free liquid films. In this case the surfaces of free liquid films are not rough at all and are also chemically homogeneous. Hence, in the case of contact angle hysteresis on free liquid films it is impossible to explain the hysteresis phenomenon by the presence of roughness and/or heterogeneity.

Earlier a qualitative theory of contact angle hysteresis of menisci in thin capillaries has been developed ${ }^{13,14}$ based on a s-shape of disjoining/conjoining pressure isotherm. The theory did not allow describing the dependency of the static advancing/receding contact angles on the capillary 
radius. Below a quantitative theory of contact angle hysteresis is presented based on the consideration of surface forces, which act in the vicinity of the three-phase contact line. This type of contact angle hysteresis exists even on a smooth, homogeneous substrate. Consideration of this kind of contact angle hysteresis on rough and/or non-homogeneous surfaces from this point of view is to be undertaken.

Evidently only a single unique value of equilibrium contact angle, $\theta_{e}$, is possible on a smooth, homogeneous surface. Hence, both static advancing $\theta_{a} \neq \theta_{e}$ and static receding $\theta_{r} \neq \theta_{e}$ contact angles as well as all contact angles in between, which are observed experimentally on such surfaces, correspond only to non-equilibrium states of the system. Hence, the picture presented by solid lines in Fig. 1 should be replaced by a new more realistic picture presented in the same picture by curve 2 .

\section{The disjoining/conjoining pressure components}

The nature of the disjoining/conjoining pressure can be briefly explained as follows. The properties of liquid in the vicinity of liquid-air and solid-liquid interfaces differ from the corresponding properties in the bulk because of surface forces action. These layers, where the surface forces act, are referred to as boundary layers (nothing to do with hydrodynamic boundary layers). In the vicinity of an apparent three phase contact line these boundary layers overlap. This overlapping of boundary layers is the reason why disjoining/conjoining pressure appears.

Contact angle hysteresis on smooth homogeneous substrates appears in the case of partial wetting, when disjoining/conjoining isotherm has a special s-shape. Components contributing to the formation of disjoining/conjoining pressure are discussed elsewhere ${ }^{15-18}$. According to DLVO theory ${ }^{17}$ these components are

1) electrostatic component, which is caused by formation of electrical double layers and their overlapping:

$\Pi_{E}=R T c_{0}(\exp (\varphi)+\exp (-\varphi))-2 R T c_{0}-\frac{(R T)^{2} \varepsilon \varepsilon_{0}}{2 F^{2}}\left(\frac{\partial \varphi}{\partial y}\right)^{2}$,

where $\mathrm{R}, \mathrm{T}, \mathrm{F}, \varepsilon, \varepsilon_{0}$ are universal gas constant, temperature in ${ }^{\circ} \mathrm{K}$; Faraday's constant, dielectric constant of water and dielectric constant of vacuum, respectively; $c_{0}$ is concentration of univalent electrolyte; $y$ and $\varphi$ are the co-ordinate normal to the liquid-air interface and dimensionless electric potential in F/RT units, respectively.

The electric potential $\varphi$ and the surface charge density $\sigma$ in Eq.(1) are related as ${ }^{17}$ 


$$
\begin{aligned}
& \sigma_{h}=\varepsilon \varepsilon_{0} \frac{R T}{F}\left(\frac{\partial \varphi}{\partial y}\right)_{y=h} \text { for the liquid/vapour interface; } \\
& \sigma_{s}=-\varepsilon \varepsilon_{0} \frac{R T}{F}\left(\frac{\partial \varphi}{\partial y}\right)_{y=0} \text { for the solid/liquid interface; }
\end{aligned}
$$

where $h$ is a separation between the interacting surfaces.

2) structural component, which is caused by water molecule dipoles orientation in a vicinity of interfaces and overlapping of these structured layers. This component is presented as a combination of both short-range and long-range interactions ${ }^{19}$ :

$\Pi_{\mathrm{S}}(h)=K_{1} \exp \left(-h / \lambda_{1}\right)+K_{2} \exp \left(-h / \lambda_{2}\right)$,

where $K_{1}, K_{2}$ and $\lambda_{1}, \lambda_{2}$ are parameters related to the magnitude and the characteristic length of the structural forces. The subscripts 1 and 2 correspond to the short-range and long-range structural interactions, respectively. Currently the latter four constants can be extracted from experimental data only. The constants $K_{1}, K_{2}$ usually have the opposite signs (see parameters values to Fig.8);

3) molecular or van der Waals component ${ }^{15,17}$ :

$\Pi_{M}(h)=\frac{A}{6 \pi h^{3}}$,

where $A=-A_{H}, A_{H}$ is the Hamaker constant. Note, the importance of the van der Waals component is usually grossly exaggerated in the literature: other components of the disjoining/conjoining pressure are equally or even more important in the case of aqueous electrolyte solutions.

The resulting disjoining/conjoining pressure isotherm has a characteristic s-shape ${ }^{17,18}$ :

$$
\Pi(h)=\Pi_{M}(h)+\Pi_{E}(h)+\Pi_{S}(h) .
$$

An example of possible shapes of the disjoining/conjoining pressure isotherms is given in Fig.2._The shape of the isotherm depends on contribution of the surface forces components and correspond to different wetting conditions.

\section{Disjoining/conjoining pressure and wetting phenomena}

Kelvin's equation describes the change in vapour pressure over the curved liquid/vapor interface (for example, a capillary or a droplet) ${ }^{20}$ :

$$
P_{e}=\frac{R T}{v_{m}} \ln \frac{p_{s}}{p}
$$


where $P_{e}=P_{v}-P_{1}$ is the excess pressure; $P_{1}$ is the pressure inside the liquid; $P_{v}$ is the pressure in the ambient vapour; $v_{m}$ is the liquid molar volume; $p_{s}$ and $p$ are the saturated vapour pressure (over a flat liquid surface) and the pressure over the curved interface, respectively.. The excess pressure inside the droplet, $P_{e}$, should be negative (pressure inside the droplet is higher than the pressure in the ambient vapour). Thus, the right-hand side of Kelvin's equation must be negative, which is possible only if $p>p_{s}$; that is, droplets can only be at equilibrium with oversaturated vapour. It is the reason why it is difficult to investigate experimentally equilibrium droplets on solid substrates: it is necessary to maintain oversaturated vapour over the substrate under investigation for a prolonged period of time ${ }^{14}$.

In contrast to a droplet equilibrium for a meniscus according to the Kelvin's equation is possible with undersaturated vapour $\left(P_{e}>0\right.$ and $\left.p<p_{s}\right)$. Note, equilibrium meniscus can exist in the case of both complete and partial wetting. The latter is different from equilibrium droplets, which can exist only in the partial wetting case; in the case of complete wetting droplets spread out completely.

Schematic presentation of two possible shapes of disjoining/conjoining pressure isotherms is given in Fig. 2. These types of dependence, $\Pi(h)$, are typical for the sum of electrostatic and van der Waals components of the disjoining/conjoining pressure (DLVO theory). Dependency 1 in Fig. 2 corresponds to the complete wetting case, while curve 2 corresponds to the partial wetting case. Note, if the structural component is taken into account then the shape of the disjoining/conjoining pressure dependency become more sophisticated ${ }^{27}$. The structural component is included below in the disjoining/conjoining pressure isotherms. However, for a case of capillary meniscus, $P_{e}>0$ and the secondary minimum of the structural interactions on isotherm has no such drastic effect on the contact angle hysteresis as in the case of droplets ${ }^{27}$.

Let us consider the conditions of equilibrium for flat wetting films in contact with a liquid meniscus or a droplet. Due to a small size of the considered systems, the gravity effect is neglected below.

In the case of complete wetting the thickness $h_{e}$ of the equilibrium wetting film corresponds to the intersection of a straight line $P_{e}>0$ with the disjoining/conjoining pressure isotherm: there is a only one intersection in the case 1 in Fig. 2.

It could be three intersections of a straight line $P_{e}>0$ (partial wetting, isotherm 2 in Fig.2) with the disjoining/conjoining pressure isotherm. However, $h_{\beta}$ and $h_{u}$ are a metastable and an unstable equilibrium thicknesses, respectively; only $h_{e}$ corresponds to a thermodynamically stable equilibrium thickness. ${ }^{14,17}$ 


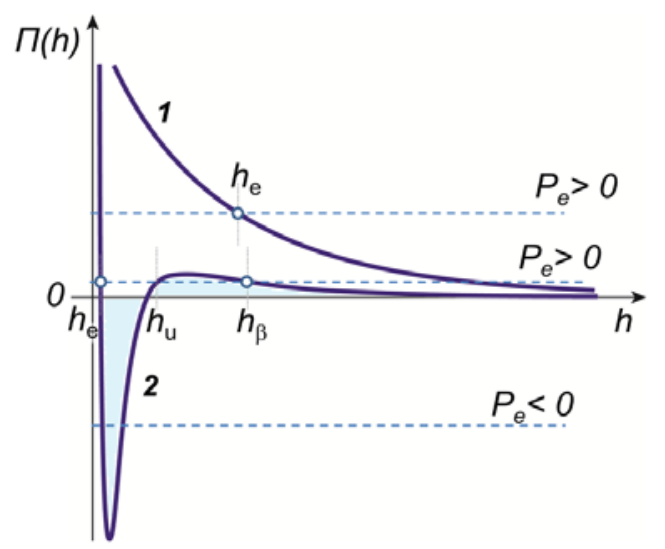

Fig. 2. Schematic presentation of disjoining/conjoining pressure isotherms.

(1) complete wetting case, (2) partial wetting case.

$h_{e}, h_{u}, h_{\beta}$-thicknesses of a stable, an unstable and a metastable wetting films respectively. ${ }^{14,17}$

The shape of the isotherms is typical for a sum of electrostatic and van der Waals interactions (DLVO theory).

The equilibrium contact angle in terms of disjoining/conjoining pressure is determined by known equation $^{17,21}$

$$
\cos \theta_{e} \approx 1+\frac{1}{\gamma} \int_{h_{e}}^{\infty} \Pi(h) d h,
$$

where $\Pi$ is the disjoining pressure; $\gamma$ is the liquid-vapour interfacial tension.

Let us discuss a two-dimensional capillary with a half-width $H$ (Fig. 3). There are two possible situations in geometrical definition of equilibrium contact angle, $\theta_{e}$ :

(i) $H<r_{e}$ (Fig. 3, case 2), this situation is referred to as partial wetting case and contact angle is defined as $\cos \theta_{e}=H / r_{e}<1$;

(ii) $H \geq r_{e}$ (Fig. 3, case 1), this situation is referred to as complete wetting; the contact angle cannot be introduced geometrically. The case of complete wetting is characterised below by the ratio $/ r_{e}$, which sometimes referred to as ' $\cos \theta_{e}>1$ '. 


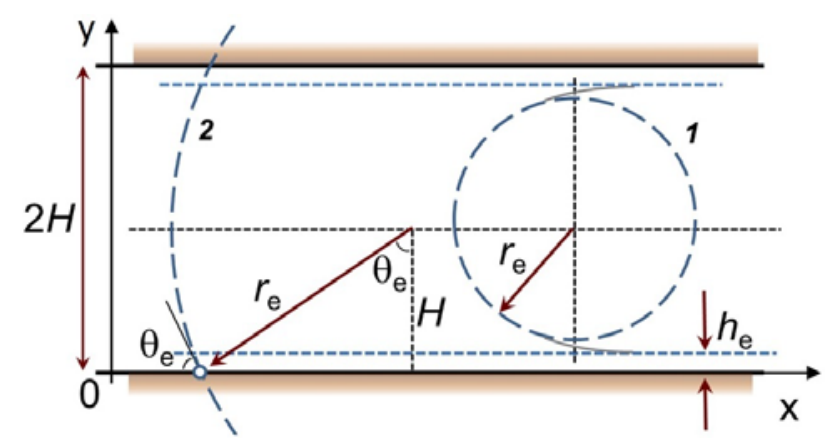

Fig. 3. A schematic presentation of two possible positions of a spherical meniscus inside two dimensional capillary.

(1) complete wetting, $r_{e} \leq H, H / r_{e} \geq 1$;

(2) partial wetting, $r_{e}>H, \cos \theta_{e}=H / r_{e}<1$.

For the complete wetting case the integral in the right-hand side in Eq.(6) is always positive ${ }^{14}$ (see curve 1 in Fig.2). For partial wetting conditions $\left(\cos \theta_{e}<1\right)$, the integral in the right hand side of Eq. (6) of the disjoining/conjoining pressure is negative (Fig.2, curve 2).

The integral $\int_{h_{e}}^{\infty} \Pi(h) d h$ can be positive even for curve 2 in Fig.2. It means that complete wetting conditions take place in this situation and $\theta_{e}=0$. The eq.(6) cannot be applied in the case of complete wetting case.

\section{Hysteresis of contact angle in capillaries}

In this section equilibrium of a meniscus in a flat capillary is briefly considered according to $13,14,22$.

If a meniscus is at equilibrium with a reservoir under the equilibrium pressure $P_{e}$, then there is no flow in the system. However, if the pressure inside the reservoir is changed by $\Delta P \neq 0$, then the flow will start immediately. In the bulk part of the meniscus a new local equilibrium can be achieved rather quickly. In this case it is possible to divide the whole system (Fig. 4) into several regions: region 1 , which is the spherical meniscus with a new radius, $r$, in a state of a new local equilibrium, a part of the transition region 2 in a state of a local equilibrium with a meniscus 1 . Inside the regions 1 and 2 the pressure is constant everywhere and equals to the new excess pressure, $P=P_{e}+\Delta P$; region 3 of a thin flat equilibrium thin film, where the pressure equals to the initial equilibrium excess pressure, $P_{e}$; transport region $2^{\prime}$ in which a viscous flow of liquid occurs and in which the pressure gradually 
changes from the value $P$ to $P_{e}$ (Fig. 4). The largest pressure drop and the higher resistance to the flow occur in the non-equilibrium part of the transition region, $2^{\prime}$, where the liquid film is very thin. Region $2^{\prime}$ covers a part of the transition region of very thin films, which immediately adjoins the equilibrium thin film.

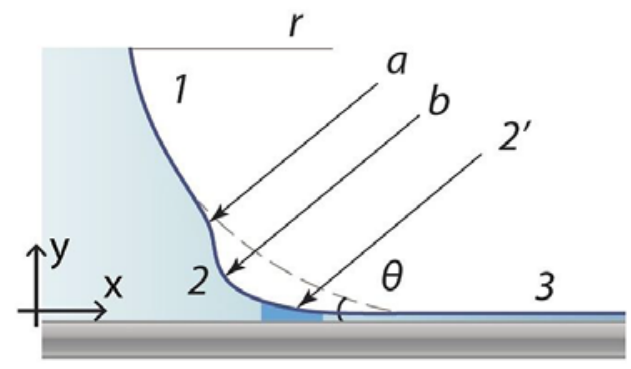

Fig. 4. The liquid profile in a capillary in the case of partial wetting in the state of local equilibrium at excess pressure $P \neq P_{e} ; r$ and $\theta$ are the radius of the spherical meniscus in the central part of the capillary and the new local equilibrium contact angle, $\theta \neq \theta_{e} .1$ - spherical meniscus of a new radius $r$, where $r \neq r_{e} ; 2$ - profile of a part of the transition zone at local equilibrium with the meniscus; 3 flat equilibrium liquid film of thickness $h_{e}$, with old equilibrium excess pressure $P_{e} ; 2$ - a flow zone inside the transition region.

Keep in mind that the capillary is in contact with the reservoir, where the pressure, $P_{a}-P_{e}-\Delta P$, is maintained, i.e., the pressure in the reservoir is lower than the atmospheric pressure, $P_{a}$.

If the pressure under the meniscus is increased then the meniscus will not move but changes its curvature to compensate for the excess pressure and, as a consequence, the contact angle increases accordingly. In this state the meniscus does not move macroscopically but it moves microscopically. This state of microscopic motion can continue for a prolong period of time if evaporation/condensation processes can be neglected. The meniscus does not move macroscopically until some critical pressure and critical contact angle, $\theta_{a}$, are reached. After further increase in pressure the flow zone occupies the region of much thicker $\beta$-films ${ }^{13,14}$ and the meniscus starts to advance macroscopically. A similar phenomenon takes place if the pressure under the meniscus is decreased: the meniscus does not recede until a critical pressure and corresponding critical contact angle, $\theta_{r}$, are reached. This means that in the whole range of contact angles, $\theta_{r}<\theta<\theta_{a}$, the meniscus does not move macroscopically but moves microscopically. 

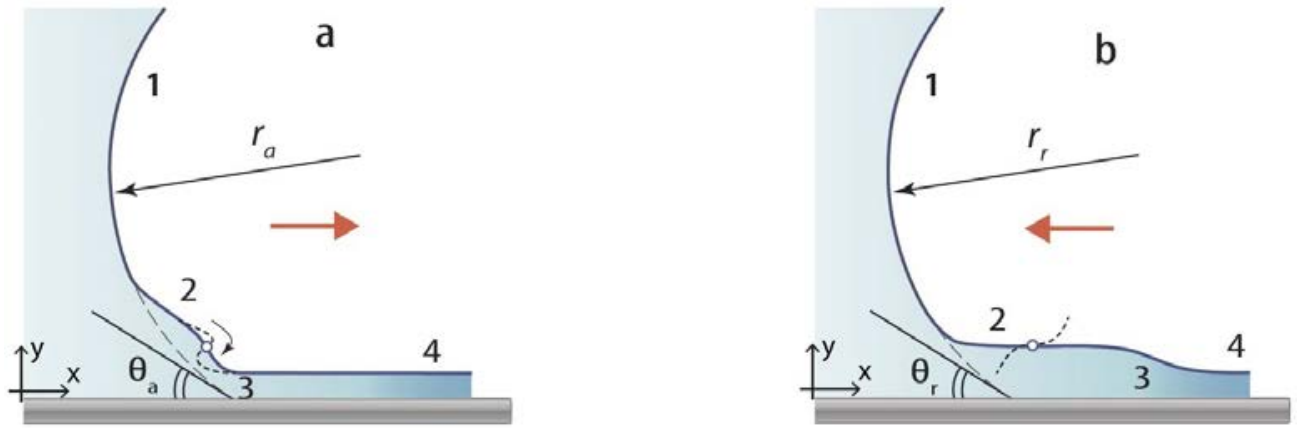

Fig. 5. Contact angle hysteresis in capillaries in the case of partial wetting (s-shaped isotherm of disjoining/conjoining pressure curve 2 in Fig.2). a - advancing contact angle: 1- a spherical meniscus of radius $r_{a}>r_{e} ; 2$ - transition zone with a "critical" marked point (see explanation in the text); 3 - flow zone; 4 - flat film. Close to the marked "critical" point a dashed line shows the profile of the transition zone just after the contact angle reaches the critical value $\theta_{a}$, the beginning of a "caterpillar motion". b - receding contact angle: 1- a spherical meniscus of radius $r_{r}<r_{e}<r_{a} ; \quad 2$ - transition zone with a "critical" marked point (see explanation in the text); 3 - flow zone, 4 - flat film. Close to the marked "critical" point the dashed line shows the profile of the transition zone just after the contact angle reaches the critical value $\theta_{a}$.

The above qualitative explanation for the contact angle hysteresis on smooth, homogeneous solid substrates is based on the s-shaped isotherm of disjoining/conjoining pressure in the case of partial wetting. The s-shaped determines a very special shape of the transition zone in the case of equilibrium meniscus (Fig. 4). In the case of increasing the pressure behind the meniscus (Fig. 5a) a detailed consideration ${ }^{13,14}$ of the transition zone shows that close to the "critical" point marked in Fig. $5 \mathrm{a}$, the slope of the profile becomes steeper with increasing pressure. In the range of very thin films (region 3 in Fig. 5a) there is a flow zone: viscous resistance in this region is very high, that is why the meniscus advances very slowly. After some critical pressure behind the meniscus is reached, then the slope at the "critical" point reaches $\pi / 2$. After that the flow step-wisely occupies the region of thick $\beta$-films the fast "caterpillar motion" starts as shown in Fig. 5 a.

In the case of decreasing the pressure behind the meniscus the event proceeds according to Fig. 5b. In this case up to some critical pressure the slope in the transition zone close to the "critical" marked point becomes more and more flat. In the range of very thin film (region 3 in Fig. $5 b$ ) there is 
a zone of flow. As in the previous case the viscous resistance in this region again is very high, that is why the receding of the meniscus proceeds very slowly. After some critical pressure behind the meniscus is reached then the profile in the vicinity of the "critical" point shows the discontinuous behavior, which is obviously impossible. This means the meniscus will start to slide along the thick $\beta$ film. This phenomenon (the presence of a thick $\beta$-film behind the receding meniscus of aqueous solutions in quartz capillaries) has been discovered experimentally ${ }^{23-24}$. This supports the presented arguments explaining static contact angle hysteresis on smooth, homogeneous substrates.

The suggested mechanism of contact angle hysteresis on smooth homogeneous surfaces has a direct experimental confirmation ${ }^{25,26}$. A qualitative estimations for advancing and receding contact angles via disjoining/conjoining pressure isotherm were deduced in ${ }^{13,14}$.

To simplify the derivation below the discussion is limited to liquids of a low volatility, whose rates of evaporation and condensation are sufficiently low. In contrast to droplets, the effect of liquid evaporation in thin capillaries is much less significant and it may be easily neglected. The main assumption is that the liquid flow from the quasi-equilibrium meniscus to the equilibrium film in front is very slow until some critical pressure difference, $\Delta P_{a}$, (in the case of advancing meniscus) or $\Delta P_{r}$ (in the case of receding meniscus) is reached. These conditions do not exist in the case of complete wetting, when the equilibrium film is sufficiently thick. However, static hysteresis is usually observed in cases of partial wetting (at $\theta>0$ ) only, when the surface of the solid body is covered with significantly thinner films, where the resistance to the viscous flow is much higher.

Two-dimensional (flat) capillary is under consideration below, so the equations includ a curvature along one direction only. The two-dimensional approach reproduces the main physical features of the system, but at the same time it makes easier the theoretical and numerical analysis of the problem. The same time the two-dimensional approach allows comparing with previous results on contact angle hysteresis in the case of two-dimensional droplers ${ }^{27}$.

The conditions are written down below for quasi-equilibrium of the meniscus in region 1 (Fig. 5). Within the region 1 all fluxes can be neglected and the excess pressure can be considered to remain constant and equal to $P=P_{e}+\Delta P=$ const. It is assumed below based on the previous consideration that for description of the quasi-equilibrium profile of the liquid, $h(x)$, in region 1 in the absence of true thermodynamic equilibrium in the whole system, the known equation ${ }^{14,17}$ for liquid profile can be used: 


$$
\frac{\gamma h^{\prime \prime}}{\left(1+h^{\prime 2}\right)^{3 / 2}}+\Pi(h)=P
$$

where the equilibrium pressure, $P_{e}$, is replaced by the new non-equilibrium pressure, $P$.

Multiplying both sides of Eq. (7) by $h^{\prime}$ and integrating it with respect to $x$ from 0 to $x$ ( $h=H$, $h^{\prime}=\infty$ at $x=0$, Fig. 3), we obtain

$$
\frac{\gamma}{\sqrt{1+h^{\prime 2}}}=\psi(h, P)
$$

where

$$
\psi(h, P)=P(H-h)-\int_{h}^{\infty} \Pi(\bar{h}) d \bar{h} .
$$

The left hand side of Eq. (8) ranges in between $0\left(\right.$ at $h^{\prime 2}=\infty$ ) and $\gamma\left(\right.$ at $h^{\prime 2}=0$ ). Hence, the same should be true for the right hand site of Eq. (8). The latter determined the region where a solution of Eq. (8) exists:

$$
0 \leq \psi(h, P) \leq \gamma
$$

There is no solution of Eq. (8) in the region where either $\psi>\gamma$ or $\psi<0$. If any of these conditions is violated then the boundary of the flow zone, and the centre of the meniscus cannot be connected by a continuous profile. Accordingly, quasi-equilibrium becomes impossible, i.e., the meniscus cannot be at quasi-equilibrium and must start moving. The violation of one of the inequalities in (10) determines the static advancing contact angle, $\theta_{a}$, and the violation of the other condition, the static receding contact angle, $\theta_{r}$.

Although the mechanism of violation of the equilibrium is understood physically, the value of the static advancing contact angle, $\theta_{a}$, cannot be calculated exactly, since the point $h=h_{1}$ (Fig. 6) belongs to a region where the liquid profile goes more abruptly and condition $\mathrm{h}^{\prime 2}<<1$ is violated. This condition is required since the disjoining/conjoining pressure, $\Pi(\mathrm{h})$ was obtained from assumption of the flat (low slope) interacting surfaces ${ }^{17}$. Such estimation of $\theta_{a}$ is given below. In the case under consideration, the value $\Delta P<0$, and the curvature of the meniscus decreases with decreasing pressure in the reservoir. It follows from Eq. (9) that for $P<P_{e}$ the curve $\psi(h, P)$ should be everywhere below the equilibrium curve $\psi\left(h, P_{e}\right)$ (Fig.6, curve 1). At $\theta=\theta_{a}$ and $P=P_{a}$ the function (9) vanishes, $\psi=0$, at $h=h_{1}$ (Fig.6, curve 1). Hence, it follows from Eq. (9) that 


$$
P_{a}\left(H-h_{1}\right)=\int_{h_{1}}^{\infty} \Pi(h) d h .
$$

The expression for the static advancing contact angle, $\theta_{a}$, in the case of capillary meniscus can be found from (11) using the general relationship $P_{a}=\gamma \cos \theta_{a} / H=\Pi\left(h_{1}\right)$ obtained from intersection of continuation of the spherical meniscus with a capillary wall:

$$
\cos \theta_{a}=\frac{1}{\gamma\left(1-\frac{h_{1}}{H}\right)} \int_{h_{1}}^{\infty} \Pi(h) d h=\frac{\Pi\left(h_{1}\right) h_{1}}{\gamma}+\frac{1}{\gamma} \int_{h_{1}}^{\infty} \Pi(h) d h .
$$

The equality $P_{a}=\Pi\left(h_{1}\right)$ is a condition of equilibrium in Fig. 6 (corresponds to the intersection point of the isotherm with a streight line $\left.\mathrm{P}_{\mathrm{a}}\right)$. The term $\frac{\Pi\left(h_{1}\right) h_{1}}{\gamma}=\frac{P_{a} h_{1}}{\gamma}$ can be obtained by substitution of $P_{a} H$ from (11) to relation: $\cos \theta_{a}=P_{a} H / \gamma$.

The functional dependence (12) coincides with the corresponding expression for the static advancing contact angle in the case of drops ${ }^{13,27}$. However, the magnitude $h_{1}$ in (12) has a different numeric value, so the static advancing contact angle for meniscus differs from the case of drops ${ }^{27}$. The dependence $\cos \theta_{a}$ on disjoining pressure allows concluding that the disjoining/conjoining isotherm determines uniquely not only the equilibrium value of the contact angle but also the static advancing contact angle, $\theta_{a}$.

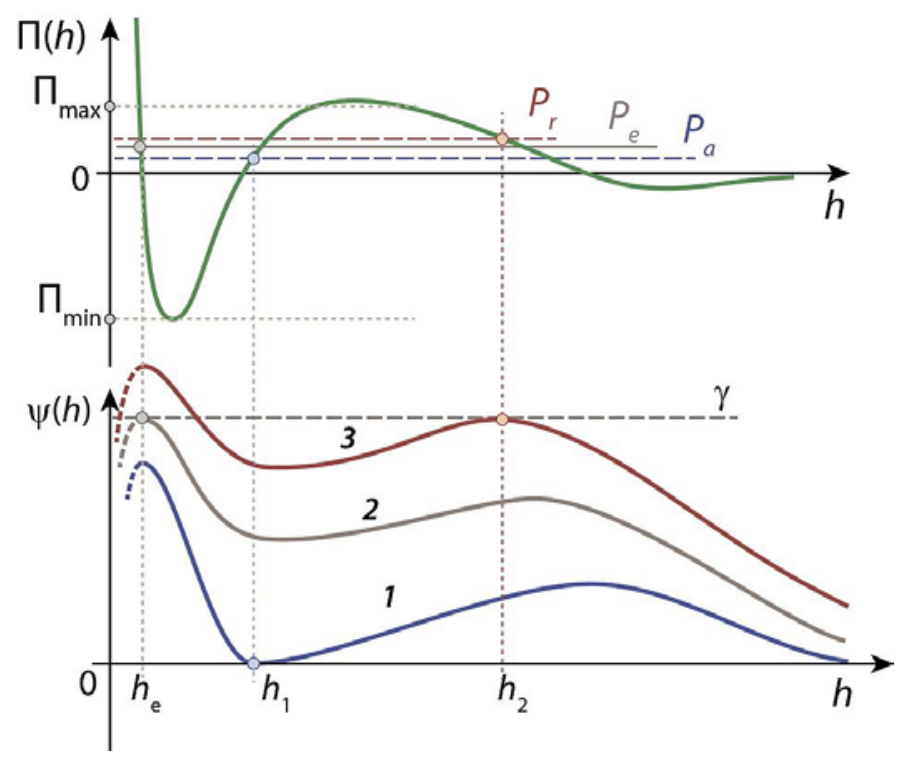


Fig. 6. Schematic plot of the function $\psi(h, P)$ according to Eq. (9) for the following cases 1advancing, 2 - equilibrium and 3 - receding.

Curve 2 in Fig. 2 touches the dashed line $\gamma$ at a single point $h_{\mathrm{e}}$. According to Eq.(8) this point corresponds to the condition $h^{\prime}=0$ which is satisfied only for a flat film at $h=h_{e}$. For any other values of $\mathrm{h}$ the liquid profile has a non-zero slope (Fig. 4).

The next step in derivation of expression for the static receding contact angle, $\theta_{r}$. In this case, the value $\Delta P>0$, since the curvature of the meniscus increases with decreasing pressure in the reservoir. It follows from Eq. (9) that for $P>P_{e}$ the curve $\psi(h, P)$ should be everywhere above the equilibrium curve $\psi\left(h, P_{e}\right)$ (Fig.6, curve 3). Violation of the conditions of quasi-equilibrium occurs in this case if $\psi(h, P)=\gamma$, i.e., an increase in $\Delta P$ to such a critical value $\Delta P_{r}$ makes the curve $\psi\left(h, P_{r}\right)$ to intersect the dashed line $\gamma=$ const .

For $P>P_{r}$, the part of the profile shown in Fig. $5 b$ by dashed line starts sliding. When the meniscus is displaced from the initial position, then a thick metastable $\beta$-film should remain behind the receding meniscus. This prediction has been confirmed experimentally ${ }^{9-11}$. The thickness of the film $h_{2}$ belongs to the $\beta$ part of the disjoining/conjoining isotherm.

Since the profile of the receding meniscus in the transition zone has a low slope, the value of the static receding contact angle, $\theta_{r}$, in the case of sufficiently thick capillaries, that is, for $P_{e}<\Pi_{\max }$ can be determined exactly. In the case of sufficiently thick capillaries, that is, for $P_{e}<\Pi_{\max }$, the substitution $\psi=\gamma$, for $P=P_{r}$ and $h=h_{2}$ into Eq. (9) gives the following equation:

$$
P_{r}\left(H-h_{2}\right)-\int_{h_{2}}^{\infty} \Pi(h) d h=\gamma,
$$

where $P_{r}=\Pi\left(h_{2}\right)$.

The static receding contact angle is obtained from Eq. (13) as

$$
\cos \theta_{r}=1+\frac{\Pi\left(h_{2}\right) h_{2}}{\gamma}+\frac{1}{\gamma} \int_{h_{2}}^{\infty} \Pi(h) d h
$$

For comparison the expression for equilibrium contact angle can be obtained as before as follows ${ }^{13}$ : 


$$
\cos \theta_{e}=1+\frac{\Pi\left(h_{e}\right) h_{e}}{\gamma}+\frac{1}{\gamma} \int_{h_{e}}^{\infty} \Pi(h) d h
$$

\section{Calculation procedure}

The above theoretical derivations have been obtained within the framework of approach to the theory of contact angle hysteresis qualitatively suggested $\mathrm{in}^{13}$. The numerical calculations and analysis based on real disjoining/conjoining pressure isotherms, comparison of the calculated data for capillary meniscus and droplet are given below for the first time.

It was explained earlier that if the contact angle $\theta$ is in between either $\theta_{e}<\theta<\theta_{a}$ or $\theta_{r}<\theta<\theta_{e}$, then the meniscus is in the state of a slow microscopic motion and ,hence, this motion can be neglected from the macroscopic point of view. It is the reason to assume that continuation of the meniscus profiles for the all three cases of advancing $(a)$, receding $(r)$ and equilibrium $(e)$ intersect the capillary wall at the same point $\mathbf{A}$ (Fig. 7). A small shift of the point $\mathbf{A}$ during transition between the states $(a),(e)$ and $(r)$ is neglected.

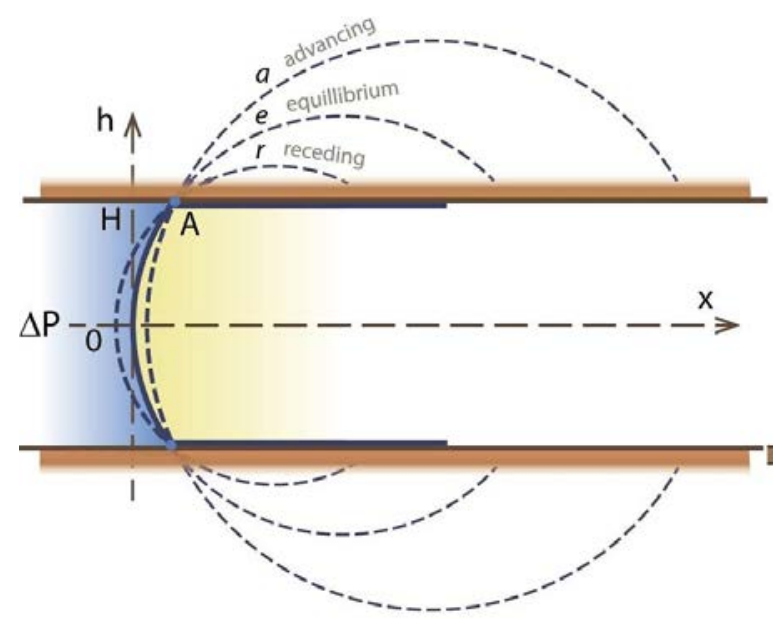

Fig. 7. The deformation of the capillary meniscus profile while changing the pressure in the reservoir by $\Delta \mathrm{P}$.

Similarly to the equilibrium meniscus, the contact angles and a capillary width are related by the following equations (see Figs. 3 and 7): 


$$
\begin{aligned}
& r_{a} \cos \theta_{a}=\frac{\gamma \cos \theta_{a}}{\Pi\left(h_{1}\right)}=H \\
& r_{r} \cos \theta_{r}=\frac{\gamma \cos \theta_{r}}{\Pi\left(h_{2}\right)}=H \\
& r_{e} \cos \theta_{e}=\frac{\gamma \cos \theta_{e}}{\Pi\left(h_{\mathrm{e}}\right)}=H
\end{aligned}
$$

The sets of equations (12),(16); (14),(17) and (15),(18) were solved.

The values $h_{e}, h_{1}, h_{2}$ were found as roots of equation: $L(h)=\frac{\gamma \cos \theta(h)}{\Pi(h)}=H$,

where $\theta_{r, e}(h)=\operatorname{acos}\left(1+\frac{\Pi(h) h}{\gamma}+\frac{1}{\gamma} \int_{h}^{\infty} \Pi(h) d h\right) ; \quad \theta_{a}(h)=\operatorname{acos}\left(\frac{\Pi(h) h}{\gamma}+\frac{1}{\gamma} \int_{h}^{\infty} \Pi(h) d h\right)$, correspondingly.

In the case of multiple solutions the roots, which satisfy the conditions (10) for $(h, P)$, were selected.

The states of equilibrium, advancing and receding correspond to the intersection points of the disjoining pressure isotherm with different straight lines of the pressures $P_{a}, P_{r}$ and $P_{e}$, respectively. The equilibrium and receding states correspond to positions of $\alpha$ - and $\beta$-films, respectively; the advancing state corresponds to intermediate (unstable) position on the isotherm.

The contact angles $\theta_{a}, \theta_{r}$ and $\theta_{e}$ were calculated at variation of the capillary width $\mathrm{H}$. The calculation results are presented in Fig. 8 a and Table 1.

The obtained data demonstrate that $\theta_{r}<\theta_{e}<\theta_{a}$, as expected. An agreement of the obtained values of contact angles values for droplets (Fig. $8 \mathrm{~b}$ ) is observed. The calculation results of advancing contact angle $\sim 57^{\circ}$, receding contact angle $\sim 9^{\circ}$ agree with calculated data for droplet ${ }^{27}$ and with experimental data ${ }^{14,16}$. Parameters of the disjoining/conjoining pressure isotherm used for these calculations are similar to that for glass surfaces ${ }^{16}$.

Table 1. The values of the advancing, equilibrium and receding contact angles as functions of the capillary size, $\mathrm{H}$.

\begin{tabular}{c|c|c|c}
\hline $\mathrm{H}, \mathrm{m}$ & $\theta_{\mathrm{a}}, \mathrm{degr}$ & $\theta_{\mathrm{e}}, \mathrm{degr}$ & $\theta_{\mathrm{r}}, \mathrm{degr}$ \\
\hline $1.0 \times 10^{-7}$ & 56.99 & 9.54 & 0 \\
\hline $5.0 \times 10^{-7}$ & 57.28 & 9.86 & 0 \\
\hline $1.0 \times 10^{-6}$ & 57.32 & 9.89 & 0 \\
\hline $5.0 \times 10^{-6}$ & 57.35 & 9.93 & 4.20 \\
\hline $1.0 \times 10^{-5}$ & 57.35 & 9.93 & 5.65 \\
\hline
\end{tabular}




\begin{tabular}{|l|l|l|l|}
\hline $5.0 \times 10^{-5}$ & 57.35 & 9.93 & 6.61 \\
\hline
\end{tabular}

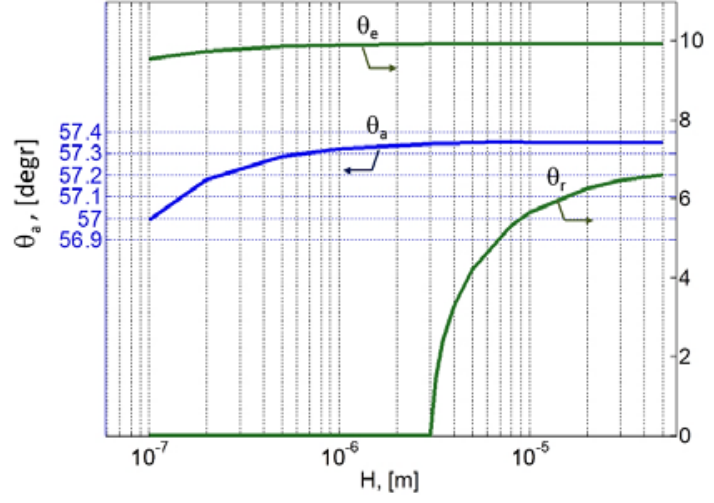

(a)

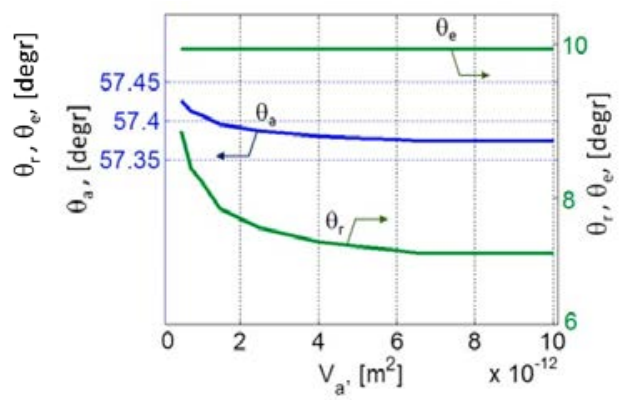

(b)

Fig.8. Dependence of the calculated values of the contact angles on the capillary width (a) and the droplet volume (b) ${ }^{27}$.

Parameters of the disjoining/conjoining pressure isotherm for both cases are identical and taken from ${ }^{27}: \sigma_{\mathrm{s}}=-150 \mathrm{mC} ; \sigma_{\mathrm{h}}=120 \mathrm{mC} ; c_{0}=1 \times 10^{-2} \mathrm{~mole} / \mathrm{m}^{3} ; A=3.5 \times 10^{-20} \mathrm{~J} ; K_{1}=2.0 \times 10^{7} \mathrm{~Pa} ; K_{2}=-$ $1 \times 10^{4} \mathrm{~Pa} ; \lambda_{1}=3.6 \times 10^{-9} \mathrm{~m} ; \lambda_{2}=26 \times 10^{-9} \mathrm{~m}$. Dilute aqueous solution of $\mathrm{NaCl}$ with the surface tension $\gamma=$ $72.7 \times 10^{-3}[\mathrm{~N} / \mathrm{m}]$ was considered. The volume unit $\left[\mathrm{m}^{2}\right]$ corresponds to the flat (two-dimesional) droplet. Arrows near the curves indicate an axis (left or right) which should be seen for this curve (two vertical axes with a different scales are used).

However, Figs. 8a and 8b show that droplets and capillaries have completely opposite behavior as a function of droplet/capillary size: the contact angles for meniscus grow with size of the capillary, whilst for the droplet, on the contrary, the bigger a droplet, the better wetting conditions (the lower the angles). This character of the contact angle dependence (Fig. 8a) in capillaries was observed experimentally in similar systems ${ }^{28}$

The dependences presented in Fig.8a and Table 1 demonstrate the interesting behaviour: in thin capillaries with low enough size, $\mathrm{H}$, a transition from partial to complete wetting occurs in the case of receding contact angle: for capillaries with $H \approx 3 \times 10^{-6} \mathrm{~m}$ the transition from partial to complete wetting is observed with a decrease in capillary width. This behaviour of the contact angles was predicted earlier ${ }^{29}$. 
The values $\theta_{r}=0$ in Table 1 were obtained when numerical calculations give $\cos \theta_{r}>1$. Mathematically this condition is senseless, but physically it corresponds to the case of compete wetting, $\theta_{r}=0$.

Transition from partial to complete wetting may be interpreted physically as a diminution of a spherical meniscus radius $r=\gamma / \mathrm{Pr}_{\mathrm{r}}$ as a result of increasing capillary pressure in small-size capillaries. This leads to receding process over thick film ( $\beta$-film) which forms from the equilibrium on the disjoining/conjoining pressure isotherm. According to experimental observations, the process of receding often goes under complete wetting conditions.

The equilibrium contact angle varies in capillaries and almost does not change in droplets, but for thick capillaries andbig droplets equilibrium contact angles coincide.

The reason of different behavior of equilibrium contact angles for droplet and capillary can be clarified based on from the analysis of the shape of the disjoining pressure isotherm.

The value $h_{e}$ (corresponding to a point of intersection between $\Pi(h)$ and $P_{e}$ ) changes very slightly at variations of $P_{e}$. This is because $\Pi(h)$ increases abruptly at lowering $h$. For droplets, $P_{e}<0$ and the integral in $\mathrm{Eq}(6)$ almost does not change with $\mathrm{P}_{\mathrm{e}}$ approaching to zero. So, the angle $\theta_{\mathrm{e}}$ for droplets changes very slightly.

For capillaries, $\mathrm{P}_{\mathrm{e}}>0$ and the integral in $\mathrm{Eq}(6)$ starts growing with $\mathrm{h}$ diminution. As a result a change in contact angle $\theta_{\mathrm{e}}$ is observed in thin capillaries.

It is important to emphasizes that for both droplets and capillaries the receding contact angles are closer to the equilibrium ones than to advancing, i.e. $\theta_{\mathrm{e}}-\theta_{\mathrm{r}} \ll \theta_{\mathrm{a}}-\theta_{\mathrm{e}}$. It is in contradiction with a well adopted view that the static advancing contact angle is a good approximation for the equilibrium contact angle. In both case droplets and capillaries (Figs. 8a and 8b) the receding contact angles are closer to the equilibrium contact angel values.

Constancy of the equilibrium contact angle of droplets can be explained based on the shape of the disjoining/conjoining pressure isotherm (see e.g. Fig. 2, curve 2 ): the value $h_{e}$ (corresponding to a point of intersection between $\Pi(h)$ and $P_{e}$ ) changes very slightly at variations of $P_{e}$. This is because $\Pi(h)$ grows very abruptly at low h. Hence, according to Eq. (6), there is a very weak dependence of the equilibrium contact angle, $\theta_{\mathrm{e}}$, on $\mathrm{P}_{\mathrm{e}}$ and, consequently, $\theta_{\mathrm{e}}$ on the droplet volume.

In capillaries the identical disjoining pressure isotherm was used as $\mathrm{in}^{27}$, however, in the case of capillaries $P_{e}>0$. It is necessary to increase $P_{e}$ rather substantially in the region of low $h$ values to 
have a small variation of $h$. This leads to a growth of positive area under the isotherm and to a decrease of the equilibrium contact angle according to Eq. (6).

For droplets the predicted dependence between the contact angles and the droplet volume was confirmed experimentally ${ }^{30,31}$. Prediction of thick $\beta$-films behind the receding meniscus in capillaries was experimentally confirmed by Churaev's group ${ }^{15,16,19}$. Presence of $\beta$-films behind the receding droplet is to be confirmed.

\section{Conclusions}

It is shown that both static advancing and receding contact angles in capillaries with smooth homogeneous walls can be calculated based on the isotherm of disjoining/conjoining pressure.

According to the theory presented both static advancing and receding contact angles are increasing function of the capillary width. . This is the opposite trend as compared with the earlier calculated static advancing and receding contact angles in the case of droplets, when the contact angles decreased with the droplet size increase. However, all three contact angles (static advancing, receding and equilibrium) coincide for big droplets and wide capillaries.

The calculation results demonstrate the effect of transition from partial to complete wetting in thin capillaries: the receding contact angle decreases to zero in this case.

\section{Acknowledgement}

This research was supported by Engineering and Physical Sciences Research Council, UK; CoWet Marie Curie ITN project, EU; COST MP1106 project and MAP EVAPORATION project, European Space Agency, COST MP 1106, EU.

\section{References}

(1) Joanny, J. F.; de Gennes, P. G. A model for contact angle hysteresis. J. Chem. Phys. 1984, 81, 552-562.

(2) Starov, V. Static contact angle hysteresis on smooth, homogeneous solid substrates. Colloid Polym Sci. 2013, 291, 261-270.

(3) Chibowski, E. Surface free energy of a solid from contact angle hysteresis. Adv. Colloid Interface Sci. 2003, 103, 149-172. 
(4) Extrand, C. W. ; Kumagai, Y. An experimental study of contact angle hysteresis. J. Colloid Interface Sci. 1997, 191, 378-383.

(5) Extrand, C. W. Water contact angle and hysteresis of polyamid surfaces. J. Colloid Interface Sci. 2002, 248, 136-142.

(6) Zorin, Z. M. ; Sobolev, V. D.; Churaev, N. V. Surface Forces in Thin Films and Disperse Systems; Nauka: Moscow, 1972; p. 214. [in Russian].

(7) Romanov, E. A.; Kokorev, D. T.; Churaev, N. V. Effect of wetting hysteresis on state of gas trapped by liquid in a capillary. Int. J. Heat Mass Transfer 1973, 16, 549-554.

(8) Rangelova, N.; Platikanov, D. Annals Univ. Sofia, Fac.Chim. 1976/1977, 71/72, 109; Platikanov, D.; Yampolskaya, G. P.; Rangelova, N.; Angarska, Zh.; Bobrova, L. E.; Izmailova, V. N. Free black films of proteins. Thermodynamic parameters. Colloid J., USSR 1981, 43, 177 180.

(9) Rangelova, N.; Platikanov, D. Annals Univ. Sofia, Fac. Chim. 1984, 78, 126.

(10) Rangelova, N. I.; Izmailova, V. N.; Platikanov, D. N.; Yampol'skaya, G. P.; Tulovskaya, S. D. Free black films of proteins: dynamic hysteresis of the contact angle (film-bulk liquid) and the rheological properties of adsorption layers. Colloid J., USSR 1990, 52, 442-447.

(11) Platikanov, D.; Nedyalkov, M.; Petkova, V. Phospholipid black foam films: dynamic contact angles and gas permeability of DMPC bilayer films. Adv. Colloid Interface Sci. 2003, 101-102, 185-203.

(12) Petkova, V.; Platikanov, D.; Nedyalkov, M. Phospholipid black foam films: dynamic contact angles and gas permeability of DMPC+DMPG black films. Adv. Colloid Interface Sci. 2003, 104, 37-51.

(13) Starov, V. M.; Velarde, M. G. Surface forces and wetting phenomena. J. Phys.: Condens. Matter 2009, 21, 464121 (11pp).

(14) Starov, V.; Velarde, M.; Radke, C. Wetting and Spreading Dynamics; Surfactant Science Series; Taylor \& Francis, 2007; Vol 138.

(15) Churaev, N. V.; Sobolev, V. D.; Starov, V. M. Disjoining Pressure of Thin Nonfreezing Interlayers. J Colloid Interface Sci. 2002, 247, 80-83.

(16) Churaev, N. V.; Sobolev, V. D. Prediction of contact angles on the basis of the FrumkinDerjaguin approach. Adv Colloid Interface Sci. 1995, 61, 1-16.

(17) Derjaguin, B. V.; Churaev, N. V.; Muller, V. M. Surface Forces; Springer, 1987. 
(18) Kuchin, I. V.; Matar, O. K.; Craster, R. V.; Starov, V. M. Influence of the disjoining pressure on the equilibrium interfacial profile in transition zone between a thin film and a capillary meniscus. Coll. Int. Sci. Comm. 2014, 1, 18-22.

(19) Churaev, N. V.; Sobolev, V. D. Wetting of low energy surfaces. Adv. Colloid Interf. Sci. 2007, 134-135, 15-23.

(20) Rowlinson, J. S. ; Widom, B. Molecular Theory of Capillarity; Clarendon Press, 1982.

(21) Frumkin, A. N. About phenomena of wetting and adhesion of bubbles. Zh. Fiz. Khim. $1938,12,337-345$.

(22) Starov, V. Static contact angle hysteresis on smooth, homogeneous solid substrates. Colloid Polym Sci. 2013, 291, 261-270.

(23) Derjaguin, B. V. ; Zorin, Z. M. Investigation of the surface condensation and vapour adsorption close to saturation conditions with micropolarization method. Zh. Fiz. Khim. 1955 $29,1755-1770$.

(24) Zorin, Z.M. ; Novikova, A.V. ; Petrov, A.K.; N.V. Churaev. Surface Forces in Thin Films and Stability of Colloids; Nauka: Moscow, 1974; p.94 [in Russian].

(25) Zorin, Z. M.; Romanov, V. P.; Churaev, N. V. The contact angles of surfactant solution on the quartz surface. Colloid \& Polymer Sci. 1979, 257, 968-972.

(26) Hayes, R. A. ; Ralston, J. Contact angle relaxation on low energy surfaces. Colloids Surfaces A. 1993, 80 (2-3), 137-146.

(27) Kuchin, I.; Starov, V. Hysteresis of Contact Angle of Sessile Droplets on Smooth Homogeneous Solid Substrates via Disjoining/Conjoining Pressure. Langmuir 2015, 31, 5345-5352.

(28) Gu, Y. Drop size dependence of contact angles of oil drops on a solid surface in water. Colloids and Surfaces A 2001, 181, 215-224.

(29) Churaev, N. V.; Setzer, M. J.; Adolphs, J. Influence of Surface Wettability on Adsorption Isotherms of Water Vapor. J. Colloid Interface Sci. 1998, 197, 327-333.

(30) Mack, G. L. The Determination of Contact Angles from Measurements of the Dimensions of Small Bubbles and Drops. I. The Spheroidal Segment Method for Acute Angles. J Phys Chem. 1936, 40 (2), 159-167.

(31) Veselovsky, V. S.; Pertsev, V. N. Adhesion of the bubbles to solid surfaces. J. Phys Chem (USSR Academy of Sciences) 1936, 8 (2), 245-259 (in Russian).

(32) Zorin, Z. M.; Romanov V. P.; Churaev, N. V. Effect of surfactants on wetting of quartz by electrolyte solutions. Kolloidn. Zh. 1979, 41(6), 1066-1073. 
(33) Martynov, G. A.; Starov, V. M.; Churaev, N. V. Hysteresis of the contact angle on homogeneous surfaces. Kolloidn. Zh. 1977, 39, 472-484.

Table of Contents/Abstract Graphic

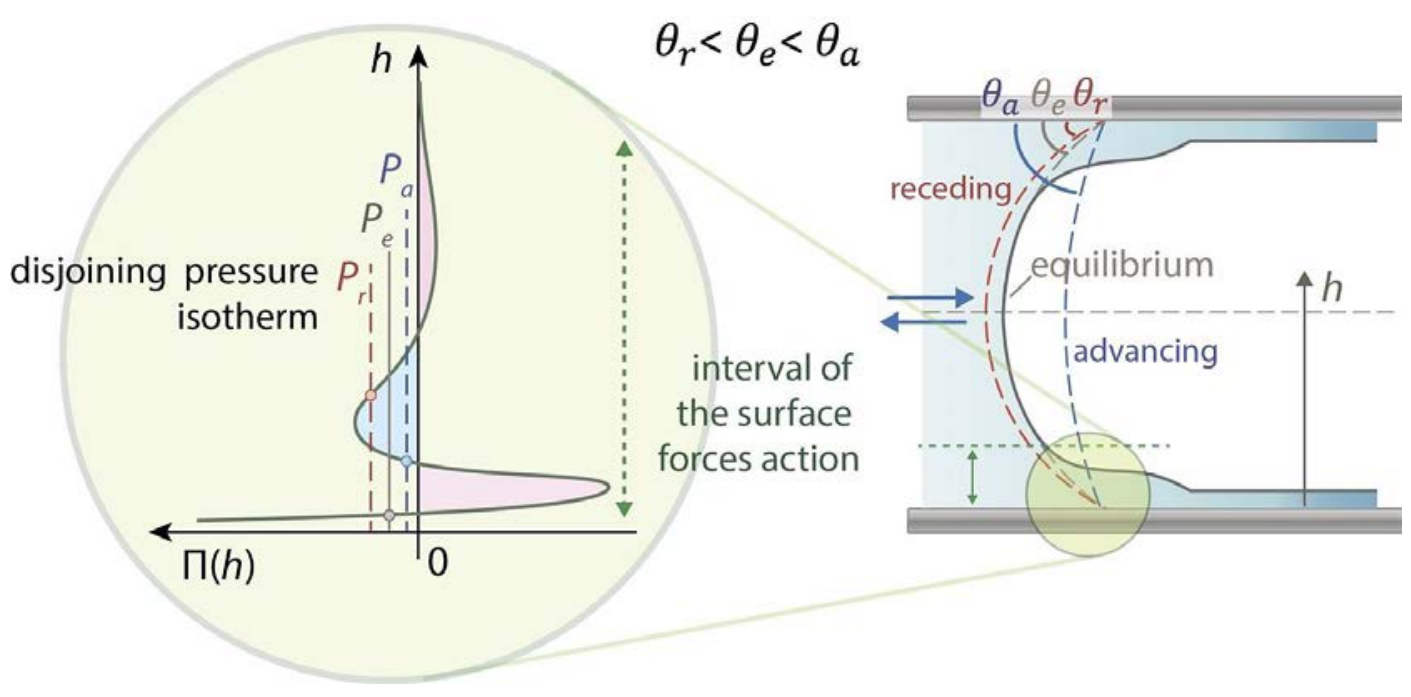

